\title{
A New Procedure for the Photometric Parallax Estimation
}

\author{
S. Karaali ${ }^{1}$, Y. Karataş, ${ }^{1}$ S. Bilir, ${ }^{1}$ S. G. Ak $^{1}$ and E. Hamzaoğlu ${ }^{2}$ \\ ${ }^{1}$ Department of Astronomy and Space Sciences, Science Faculty, Istanbul University, 34452 Istanbul, Turkey \\ karsa@istanbul.edu.tr \\ 2 Faculty of Engineering and Design, Istanbul Commerce University, 34378 Istanbul, Turkey \\ Received 2003 March 20, accepted 2003 June 19
}

\begin{abstract}
We present a new procedure for photometric parallax estimation. The data for 1236 stars provide calibrations between the absolute magnitude offset from the Hyades main-sequence and the ultravioletexcess for eight different $(B-V)_{0}$ colour-index intervals, $(0.30 .4),(0.40 .5),(0.50 .6),(0.60 .7),(0.70 .8)$,

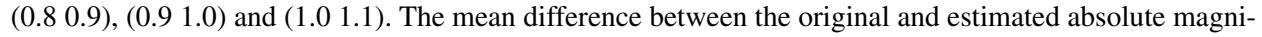
tudes and the corresponding standard deviation are rather small, +0.0002 and $\pm 0.0613 \mathrm{mag}$. The procedure has been adapted to the Sloan photometry by means of colour equations and applied to a set of artificial stars with different metallicities. The comparison of the absolute magnitudes estimated by the new procedure and the canonical one indicates that a single colour-magnitude diagram does not supply reliable absolute magnitudes for stars with large range of metallicity.
\end{abstract}

Keywords: photometric parallax — UBV photometry — Sloan photometry

\section{Introduction}

Stellar kinematics and metallicity are two primary means to deduce the history of our Galaxy. However, such goals can not be achieved without stellar distances. The distance to a star can be evaluated by trigonometric or photometric parallaxes. Trigonometric parallaxes are only available for nearby stars where Hipparcos (ESA, 1997) is the main supplier for the data. For stars at large distances, the use of photometric parallaxes is unavoidable. In other words the study of the Galactic structure is strictly tied to precise determination of absolute magnitudes.

Different methods can be used for absolute magnitude determination. The method used in the Strömgren's $u v b y$ $\beta$ (Nissen \& Schuster 1991) and in the UBV (Laird et al. 1988 , hereafter LCL) photometry depends on the absolute magnitude offset from a standard main-sequence. In recent years the derivation of absolute magnitudes has been carried out by means of colour-absolute magnitude diagrams of some specific clusters whose metal abundances are generally adopted as the mean metal abundance of a Galactic population, such as thin, thick disks and halos. The studies of Phleps et al. (2000) and Chen et al. (2001) can be given as examples. A slightly different approach is that of Siegel et al. (2002) where two relations, one for stars with solar-like abundances and another one for the metalpoor stars were derived between $M_{\mathrm{R}}$ and the colour-index $R-I$, where $M_{\mathrm{R}}$ is the absolute magnitude in the $R$ filter of Johnson system. For a star of given metallicity and colour, absolute magnitude can be estimated by linear interpolation of two ridgelines and by means of linear extrapolation beyond the metal-poor ridgeline.

We strongly believe we can contribute to this important topic by modifying the method of LCL and by adapting it to Sloan photometry. LCL used the equation

$$
M_{\mathrm{V}}(\text { Hyades })=5.64(B-V)_{0}+1.11
$$

for the fiducial main-sequence of Hyades as a standard main-sequence and derived the metallicity-dependent offset

$$
\begin{aligned}
\Delta M_{\mathrm{V}}^{\mathrm{H}}= & \frac{2.31-1.04(B-V)_{0}}{1.594} \\
& \times\left(-0.6888 \delta+53.14 \delta^{2}-97.004 \delta^{3}\right)
\end{aligned}
$$

LCL state that the calibration is valid for $\delta \leq 0.25$, which is equal to $[\mathrm{Fe} / \mathrm{H}]=-1.75 \mathrm{dex}$, according to the Carney (1979) transformation of $\delta$ into $[\mathrm{Fe} / \mathrm{H}]$

$$
[\mathrm{Fe} / \mathrm{H}]=0.11-2.90 \delta-18.68 \delta^{2}
$$

Moreover, LCL give an equation for direct absolute magnitude derivation for extreme metal-poor stars

$$
M_{\mathrm{V}}(B-V)=4.60(B-V)_{0}+3.46+1.67(\delta-0.25)
$$

As these equations reveal, the method of LCL is based on the fact that absolute magnitude (and metallicity) is a function of UV-excess, in addition to colour-index. UVexcess is usually defined as the de-reddened $(U-B)$ colourindex difference between a star and a Hyades star of equal $(B-V)_{0}$. The $U$-band is centred at a wavelength where metallicity effect is efficient, hence a star with bright $U$-magnitude, i.e. a relatively metal-poor star, is absolutely faint relative to a Hyades star of equal $(B-V)_{0}$.

We considered the possibility of calibrating the absolute magnitude offset from the updated Hyades sequence (derivation given in full in the Accessory Material)

$$
\begin{aligned}
M_{\mathrm{V}}(\text { Hyades })= & -1.48739(B-V)_{0}^{2} \\
& +7.70982(B-V)_{0}+0.331195
\end{aligned}
$$

using only $\delta$ for different $(B-V)_{0}$ colour-indices without any restriction for metallicity. This is the main scope of this work. We will show in the following sections that such an approach provides more precise absolute magnitudes than those of LCL. In Section 2 we present the data used 
for calibration and in Section 3 the procedure used for calibration is given. The extension of this procedure to the Sloan photometry is given in Section 4 and in Section 5 a detailed discussion is provided.

\section{The Data}

The $V, B-V, U-B$ and $E(B-V)$ photometric data used in this paper and the star distance $d$ are taken from Ryan (1989). For any star the following reductions have been applied

$$
\begin{aligned}
(B-V)_{0}= & (B-V)-E(B-V) \\
(U-B)_{0}= & (U-B)-0.72 E(B-V) \\
& +0.05 E^{2}(B-V) \\
M_{\mathrm{V}}= & V-3.1 E(B-V)+5-5 \log d \\
\delta(U-B)= & (U-B)_{\mathrm{H}}-(U-B)_{0}
\end{aligned}
$$

$(U-B)_{\mathrm{H}}$ is the de-reddened $(U-B)$ colour-index of a dwarf star of the Hyades cluster with the same $(B-V)_{0}$ of the star considered. We indicate with $\Delta M_{\mathrm{V}}^{\mathrm{H}}$ the absolute magnitude difference between a star and a Hyades star of equal $(B-V)_{0}$ and with $\delta_{0.6}$ the normalised UVexcess of the star considered (see Table 1 ), namely $\delta_{0.6}$ is the de-reddened $(U-B)$ colour-index difference between two stars just quoted and necessary coefficient used here is given by Sandage (1969). This procedure is necessary for the equivalence of UV-excess of two stars of the same metal-abundance, one with any $(B-V)_{0}$ and another one with $(B-V)_{0}=0.6$, where the latter is adopted as a reference colour-index for this reduction (Sandage, 1969). Contrary to Laird et al. (1988) who gave relations as a function of both colour-index $(B-V)_{0}$ and $\delta_{0.6}$ (equation 2) we prefer to plot $\Delta M_{\mathrm{V}}^{\mathrm{H}}$ as a function of only $\delta_{0.6}$ for different $(B-V)_{0}$ intervals, $0.3<$ $(B-V)_{0} \leq 0.4 ; 0.4<(B-V)_{0} \leq 0.5 ; 0.5<(B-V)_{0} \leq 0.6$; $0.6<(B-V)_{0} \leq 0.7 ; 0.7<(B-V)_{0} \leq 0.8 ; 0.8<(B-V)_{0} \leq$ $0.9 ; 0.9<(B-V)_{0} \leq 1.0$ and $1.0<(B-V)_{0} \leq 1.1$. This approach significantly improves the calibrations with respect to those of LCL, as explained in the following sections.

\section{Photometric Parallaxes}

\subsection{Calibration of Absolute Magnitude as a Function of $U V$-Excess}

We used $\Delta M_{\mathrm{V}}^{\mathrm{H}}=M_{\mathrm{V}}\left(^{*}\right)-M_{\mathrm{V}}(H)$ and the $\delta_{0.6}$ data listed in Table $\mathrm{S} 1$ for a third-degree polynomial, fitting for each $(B-V)_{0}$ interval cited in Section 2, where $M_{\mathrm{V}}(H)$ and $M_{\mathrm{V}}\left(^{*}\right)$ are the absolute magnitudes of a Hyades star, evaluated by equation $\left(1^{\prime}\right)$, and of a programme star of equal $(B-V)_{0}$, respectively. Stars are separated into different bins in $\delta_{0.6}$ with range $\Delta \delta_{0.6}=0.05 \mathrm{mag}$ in order to take into account all the programme stars and to provide reliable statistics. The number of bins is 6 for the bluest and reddest intervals of $0.3<(B-V)_{0} \leq 0.4$ and $1.0<(B-V)_{0} \leq$ 1.1 and lie between 9 and 12 for the other six colourindex intervals. The mean of $\delta_{0.6}$ and $\Delta M_{\mathrm{V}}^{\mathrm{H}}$ are evaluated for each bin except one bin in each interval of $0.7<$ $(B-V)_{0} \leq 0.8 ; \quad 0.8<(B-V)_{0} \leq 0.9 ; \quad 0.9<(B-V)_{0} \leq 1.0$ and $1.0<(B-V)_{0} \leq 1.1$, which have relatively extreme $\delta_{0.6}$ or $\Delta M_{\mathrm{V}}^{\mathrm{H}}$ values. According to this criterion, eight stars were excluded from the analysis (see Table 1 ). Then $\Delta M_{\mathrm{V}}^{\mathrm{H}}$ was plotted versus $\delta_{0.6}$ (Fig. 1) and a third-degree polynomial was fitted for each set of data

$$
\Delta M_{\mathrm{V}}^{\mathrm{H}}=a_{3} \delta_{0.6}^{3}+a_{2} \delta_{0.6}^{2}+a_{1} \delta_{0.6}+a_{0}
$$

The coefficients, $a_{i}$, of this equation are given in Table 2 as a function of $(B-V)_{0}$. One notices two important points in Figure 1. First, a large scattering between the curves exists and, second, contrary to expectations, neither of the curves converge towards the origin. This means that a star with $\delta_{0.6}=0$, corresponding to the absolute magnitude of a Hyades star, would have a value for $\Delta M_{\mathrm{V}}^{\mathrm{H}}$ different from zero and, hence, a different absolute magnitude from the Hyades star, according to the definition of $\Delta M_{\mathrm{V}}^{\mathrm{H}}$. This is a contradiction. The curves should pass through the origin to avoid this discrepancy. Table 2 shows that all the zero points are larger than $\Delta M_{\mathrm{V}}^{\mathrm{H}}=0.2 . \Delta M_{\mathrm{V}}^{\mathrm{H}}$ increases from $\Delta M_{\mathrm{V}}^{\mathrm{H}}=0.24$ for the interval $0.3<$ $(B-V)_{0} \leq 0.4$ to a maximum value of $\Delta M_{\mathrm{V}}^{\mathrm{H}}=0.35$ in the interval $0.6<(B-V)_{0} \leq 0.7$ and declines to such a lower value as $\Delta M_{\mathrm{V}}^{\mathrm{H}}=0.29$ for the interval $1.0<(B-V)_{0} \leq 1.1$.

We would like to quote the work of Cameron (1985), who discussed the same relation, i.e. $\Delta M(V)$ versus $\delta_{0.6}$. We fitted a third degree polynomial for his data (Table 2 and Figure 6 in that paper) with a constant term of -0.1663 , which is absolutely equal and almost half of the mean of the constant terms in our work. The work of Cameron (1985) also indicates that $\delta_{0.6}=0$ does not imply $\Delta M(V)=0.0$.

\subsection{Normalisation of the Hyades Main Sequence}

The discrepancy mentioned above can be minimised by normalisation of the Hyades main-sequence. In other words, $M_{\mathrm{V}}^{\mathrm{H}}$ needs to be incremented to limit the constant term in equation (6). Table 3 gives $M_{\mathrm{V}}^{\mathrm{H}}$ as evaluated by equation $\left(1^{\prime}\right)$, and the adopted $M_{\mathrm{V}}^{\mathrm{H}}$, i.e. $M_{\mathrm{V}}^{\mathrm{H}}(\mathrm{ad})=M_{\mathrm{V}}^{\mathrm{H}}(\mathrm{ev})+a_{0}$, where $a_{0}$ is the corresponding zero point in equation (6). The $M_{\mathrm{V}}^{\mathrm{H}}(\mathrm{ad})$ are plotted against the mean $(B-V)_{0}$ for each interval and the following quadratic equation has been fitted (Figure 2).

$$
M_{\mathrm{V}}^{\mathrm{H}}(\text { nor })=-2.1328(B-V)_{0}^{2}+8.6803(B-V)_{0}+0.305
$$

This is the normalised colour-magnitude equation for the Hyades main-sequence used in the derivation of photometric parallaxes.

\subsection{Final Equations for Photometric Parallaxes}

After normalisation, the difference in absolute magnitude between a star and a Hyades star of equal $(B-V)_{0}$, i.e. $\Delta M_{\mathrm{V}}^{\mathrm{H}}$ (nor) is re-evaluated and used in final equations for photometric parallaxes (see Table S2). The procedure is 
Table 1. Normalised UV-excesses $\left(\delta_{0.6}\right)$ and two sets of absolute magnitude differences $\left(<\Delta \mathbf{M}_{1}>\equiv \Delta M_{\mathrm{V}}^{\mathrm{H}}\right.$ and $<\Delta \mathrm{M}_{2}>\equiv \Delta M_{\mathrm{V}}^{\mathrm{H}}$ (nor)), in different bins for stars in eight $(B-V)_{0}$ colour index intervals. $N$ is the total number of stars in each bin

\begin{tabular}{lllllllll}
\hline$\delta_{0.6}$-interval & $<\delta_{0.6}>$ & $<\Delta M_{1}>$ & $<\Delta M_{2}>$ & $N$ & $\delta_{0.6}$-interval & $<\delta_{0.6}>$ & $<\Delta M_{1}>$
\end{tabular}

(a) $0.3<(B-V)_{0} \leq 0.4$

$(+0.025+0.075] \quad 0.045$

$(+0.175+0.225] \quad 0.200$

$(+0.225+0.275]$

$(+0.275+0.325]$

$(+0.325+0.375]$

0.260

0.304

0.340

\subsection{3}

0.364

0.907

1.249

1.332

1.384

(b) $0.4<(B-V)_{0} \leq 0.5$

$(-0.125-0.075] \quad-0.110$

$(-0.075-0.025]-0.10$

$(-0.025+0.025] \quad-0.030$

$\begin{array}{ll}(+0.025+0.075] & 0.055\end{array}$

$(+0.075+0.125] \quad 0.096$

$(+0.125+0.175] \quad 0.159$

$(+0.175+0.225] \quad 0.204$

$(+0.225+0.275]$

$(+0.275+0.325]$

$(+0.325+0.375]$

0.250

0.301

0.339

$(+0.375+0.425]$

0.390

(c) $0.5<(B-V)_{0} \leq 0.6$

$(-0.075-0.025]$

$(-0.025+0.025]$

$-0.060$

$(+0.025+0.075]$

$(+0.075+0.125]$

$(+0.125+0.175]$

$(+0.175+0.225]$

$(+0.225+0.275]$

$(+0.275+0.325]$

$(+0.325+0.375]$

\subsection{5}

0.053

0.104

0.153

0.202

0.250

0.292

0.337

$$
\begin{array}{r}
-0.121 \\
0.097 \\
0.290 \\
0.552 \\
0.750 \\
1.102 \\
1.230 \\
1.360 \\
1.525 \\
1.609 \\
1.698
\end{array}
$$

-0.393
-0.197
0.006
0.269
0.469
0.814
0.947
1.078
1.224
1.330
1.435

-0.046
0.115
0.660
0.992
1.081
1.137

1
2
2
4
25
11

(d) $0.6<(B-V)_{0} \leq 0.7$

$(-0.175-0.075]$
$(-0.075-0.025]$
$(-0.025+0.025]$
$(+0.025+0.075]$
$(+0.075+0.125]$
$(+0.125+0.175]$
$(+0.175+0.225]$
$(+0.225+0.275]$
$(+0.275+0.325]$
$(+0.325+0.425]$

$-0.153$

$-0.028$

0.013

0.052

0.102

0.150

0.202

0.250

0.297

0.353 (e) $0.7<(B-V)_{0} \leq 0.8$

$\begin{array}{rrr}(-0.200-0.125] & - & - \\ (-0.125-0.075] & -0.089 & -0.104 \\ (-0.075-0.025] & -0.041 & 0.100 \\ (-0.025+0.025] & -0.001 & 0.328 \\ (+0.025+0.075] & 0.052 & 0.568 \\ (+0.075+0.125] & 0.102 & 0.744 \\ (+0.125+0.175] & 0.145 & 0.923 \\ (+0.175+0.225] & 0.196 & 1.117 \\ (+0.225+0.275] & 0.247 & 1.207 \\ (+0.275+0.325] & 0.297 & 1.395 \\ (+0.325+0.375] & 0.345 & 1.491\end{array}$

$\begin{array}{rr}-\bar{r} & 2 \\ -0.442 & 8 \\ -0.238 & 11 \\ -0.010 & 34 \\ 0.230 & 47 \\ 0.405 & 46 \\ 0.585 & 30 \\ 0.778 & 15 \\ 0.869 & 9 \\ 1.057 & 7 \\ 1.153 & 8\end{array}$

(f) $0.8<(B-V)_{0} \leq 0.9$

$\begin{array}{crrrr}(-0.175-0.125] & -0.154 & -0.228 & -0.561 & 5 \\ (-0.125-0.075] & -0.093 & 0.020 & -0.309 & 14 \\ (-0.075-0.025] & -0.050 & 0.144 & -0.186 & 22 \\ (-0.025+0.025] & 0.000 & 0.323 & -0.009 & 27 \\ (+0.025+0.075] & 0.046 & 0.450 & 0.118 & 26 \\ (+0.075+0.125] & 0.103 & 0.657 & 0.325 & 21 \\ (+0.125+0.175] & 0.145 & 0.813 & 0.482 & 13 \\ (+0.175+0.225] & 0.200 & 0.947 & 0.617 & 5 \\ (+0.225+0.275] & 0.255 & 1.201 & 0.867 & 8 \\ (+0.275+0.325] & 0.299 & 1.261 & 0.929 & 8 \\ (+0.325+0.375] & 0.345 & 1.482 & 1.148 & 4 \\ (+0.375+0.425] & 0.398 & 1.525 & 1.196 & 4 \\ (+0.425+0.600] & - & - & - & 4\end{array}$

(g) $0.9<(B-V)_{0} \leq 1.0$

$(-0.075-0.025] \quad-0.055$

$(-0.025+0.025] \quad 0.004$

$(+0.025+0.075] \quad 0.058$

$(+0.075+0.125] \quad 0.105$

$(+0.125+0.175]$

$(+0.175+0.225]$

0.151

0.198

$(+0.275+0.325]$

0.300

0.226

$-0.081$

$0.334 \quad 0.017$

$0.452 \quad 0.137$

$\begin{array}{ll}0.585 & 0.272\end{array}$

$0.706 \quad 0.392$

$\begin{array}{ll}0.811 & 0.498\end{array}$

(h) $1.0<(B-V)_{0} \leq 1.1$

$(-0.325-0.275]$

$(+0.025+0.075]$

$(+0.075+0.125]$

$(+0.125+0.175]$

$(+0.175+0.225]$

$(+0.225+0.275]$

$(+0.275+0.325]$

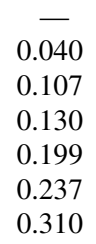

$-\overline{336}$
0.478
0.489
0.600
0.754
0.853 $-\overline{-}$
0.047
0.195
0.204
0.323
0.469
0.572
11

34

47

0

$N$

(n)

8


Table 2. Numerical values for the coefficients in equation (6) as a function of $(B-V)_{0}$ colour-index

\begin{tabular}{|c|c|c|c|c|}
\hline$(B-V)_{0}$ & $a_{3}$ & $a_{2}$ & $a_{1}$ & $a_{0}$ \\
\hline (0.3 0.4$]$ & -35.7800 & +17.9170 & +1.4505 & +0.2389 \\
\hline (0.4 0.5] & -15.4620 & +3.5129 & +4.5340 & +0.2865 \\
\hline (0.5 0.6] & +4.9011 & -5.3226 & +5.4334 & +0.3294 \\
\hline (0.6 0.7 0.7 & -11.0040 & -0.3570 & +5.0207 & +0.3491 \\
\hline (0.7 0.8 ] & +0.0737 & -3.6154 & +4.6223 & +0.3237 \\
\hline (0.8 0.9] & -2.4661 & +0.1822 & +3.4514 & +0.3102 \\
\hline (0.9 1.0] & -20.8350 & +6.0860 & +2.0942 & +0.3206 \\
\hline (1.0 1.1] & -8.3965 & +5.0002 & +1.0912 & +0.2903 \\
\hline
\end{tabular}

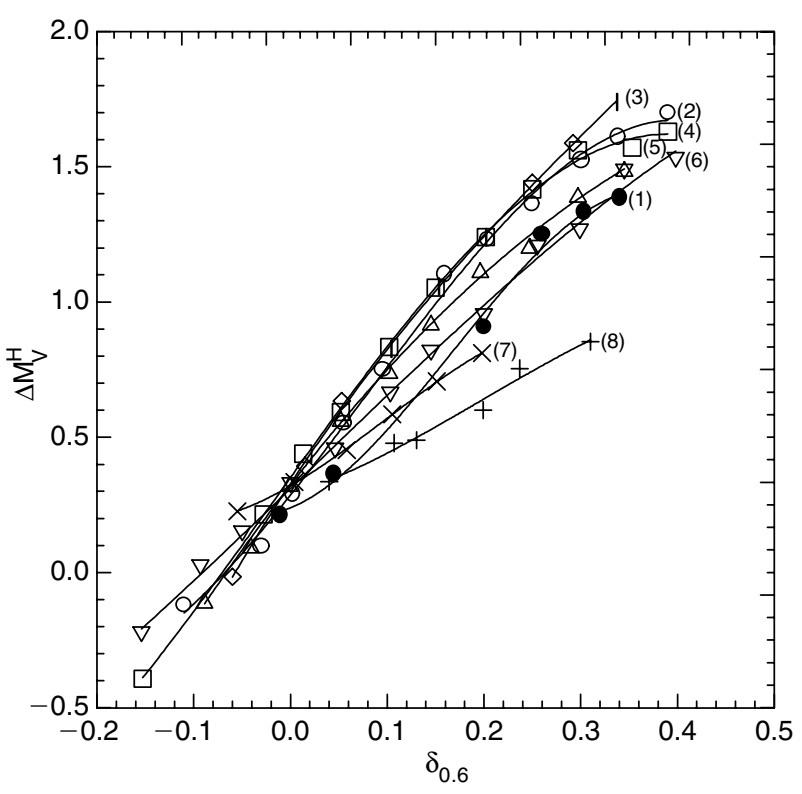

Figure $1 \Delta M_{\mathrm{V}}^{\mathrm{H}}$ versus $\delta_{0.6}$ for eight $(B-V)_{0}$ colour-index intervals. The symbols are $(\bullet): 0.3<(B-V)_{0} \leq 0.4 ; \quad(O)$ : $0.4<(B-V)_{0} \leq 0.5 ;(\diamond): 0.5<(B-V)_{0} \leq 0.6 ;(\square): 0.6<(B-V)_{0} \leq$ $0.7 ;(\Delta): 0.7<(B-V)_{0} \leq 0.8 ;(\nabla): 0.8<(B-V)_{0} \leq 0.9 ;(\times): 0.9<$ $(B-V)_{0} \leq 1.0$; and $(+): 1.0<(B-V)_{0} \leq 1.1$.

Table 3. Two sets of absolute magnitudes for the Hyades cluster as a function of $(B-V)_{0}$ colour-index. $M_{\mathrm{V}}^{\mathrm{H}}$ evaluated by equation $\left(1^{\prime}\right)$ and $M_{V}^{H}(\mathrm{ad})$ adopted for normalisation

\begin{tabular}{|c|c|c|c|}
\hline$(B-V)_{0}$ & $<(B-V)_{0}>$ & $M_{\mathrm{V}}^{\mathrm{H}}$ & $M_{\mathrm{V}}^{\mathrm{H}}(\mathrm{ad})$ \\
\hline (0.3 0.4$]$ & 0.384 & 3.07 & 3.31 \\
\hline (0.4 0.5$]$ & 0.458 & 3.55 & 3.84 \\
\hline$\left(\begin{array}{ll}0.5 & 0.6\end{array}\right]$ & 0.557 & 4.16 & 4.49 \\
\hline (0.6 0.7 ] & 0.655 & 4.74 & 5.09 \\
\hline (0.7 0.8$]$ & 0.751 & 5.28 & 5.61 \\
\hline (0.8 0.9 ] & 0.854 & 5.83 & 6.14 \\
\hline (0.9 1.0] & 0.945 & 6.29 & 6.61 \\
\hline (1.0 1.1] & 1.045 & 6.76 & 7.05 \\
\hline
\end{tabular}

and their standard deviations are only few percent. However, this is not the result for the procedure applied by LCL (Table 5 and Figure 4; see Section 5 for detailed discussion).

The evolutionary effect has not been considered above. However, the $(U-B)_{0}$ versus $(B-V)_{0}$ sequence slightly

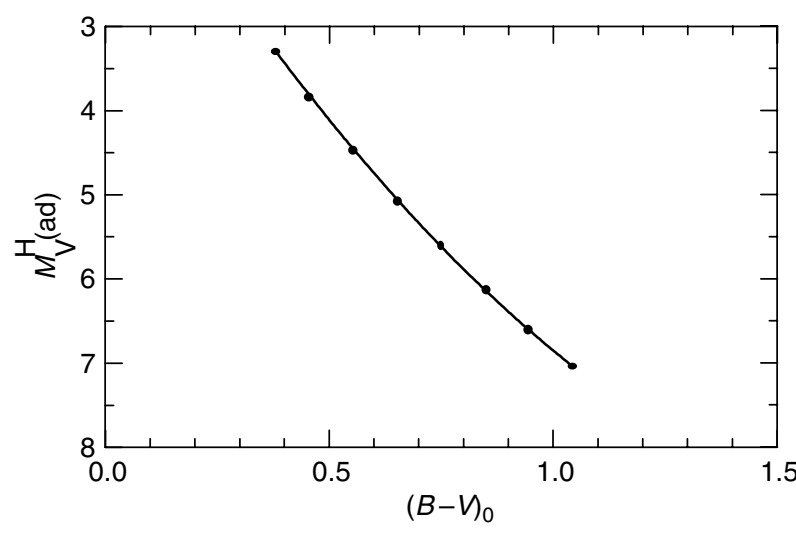

Figure 2 Adopted absolute magnitudes for the Hyades main-sequence versus $(B-V)_{0}$ colour-index.

Table 4. Numerical values for the coefficients of equation (8) as a function of $(B-V)_{0}$ colour-index

\begin{tabular}{|c|c|c|c|c|}
\hline$(B-V)_{0}$ & $b_{3}$ & $b_{2}$ & $b_{1}$ & $b_{0}$ \\
\hline ( $\left.\begin{array}{ll}0.3 & 0.4\end{array}\right]$ & -32.1800 & +15.9370 & +1.7350 & -0.0177 \\
\hline (0.4 0.5$]$ & -15.3820 & +3.7188 & +4.4850 & +0.0022 \\
\hline (0.5 0.6$]$ & +3.9109 & -4.8075 & +5.3847 & +0.0134 \\
\hline (0.6 0.7 0.7 & -11.1700 & -0.3015 & +5.0281 & +0.0153 \\
\hline (0.7 0.8 ] & +0.1049 & -3.6157 & +4.6196 & -0.0144 \\
\hline (0.8 0.9 (0.9 & -22.5350 & +0.1109 & +3.4469 & -0.0203 \\
\hline (0.9 1.0$]$ & -24.9710 & +7.2916 & +2.0269 & +0.0051 \\
\hline (1.0 1.1] & -7.4029 & +4.2761 & +1.2638 & -0.0047 \\
\hline
\end{tabular}

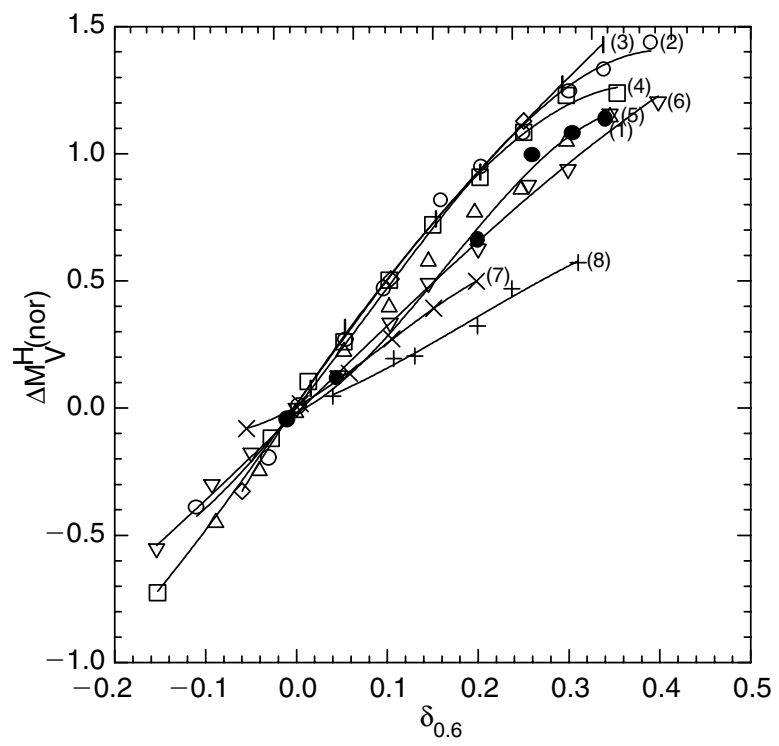

Figure $3 \quad \Delta M_{\mathrm{V}}^{\mathrm{H}}$ (nor) versus $\delta_{0.6}$ for eight $(B-V)_{0}$ colour-index intervals (symbols as in Figure 1).

varies as a function of the gravity. Therefore for stars close to the end of the main sequence (TAMS), the estimate of real $\delta_{0.6}$ is smaller. We used the Yale isochrones of Yi et al. (2001) for the following chemical composition and checked the size of the errors introduced by evolutionary effects between the zero age main-sequence (ZAMS) and TAMS (10 Gyr): $Y=0.27$ and $Z=0.02$ 
$([\mathrm{Fe} / \mathrm{H}]=+0.05 \mathrm{dex})$. For this sample, $<\delta_{0.6}>=0.0$ and 0.02 for $0.81<(B-V)_{0} \leq 1.00$ and $0.7<(B-V)_{0} \leq 0.81$, respectively. The effect of the difference in $\Delta M_{\mathrm{V}}^{\mathrm{H}}$ (nor) for a star with $\delta_{0.6}=0.2([\mathrm{Fe} / \mathrm{H}]=-1.2 \mathrm{dex})$ is $8 \%$.

\section{Extension of the Procedure to the Sloan Photometry}

\subsection{Transformation of the Normalised UV-Excess from UBV to the Sloan Photometry and New Metallicity Calibration}

The following colour equations of Fukugita et al. (1996) provide a relation between the normalised UV-excesses

Table 5. The mean difference between the original absolute magnitudes and the absolute magnitudes estimated by two different procedures and the corresponding standard deviations

\begin{tabular}{|c|c|c|c|c|}
\hline \multirow[t]{2}{*}{$(B-V)_{0}$} & \multicolumn{2}{|c|}{$<M_{V}($ ori $)-M_{V}($ est $)>$} & \multicolumn{2}{|c|}{$\sigma$} \\
\hline & $\begin{array}{l}\text { New } \\
\text { procedure }\end{array}$ & LCL & $\begin{array}{l}\text { New } \\
\text { procedure }\end{array}$ & LCL \\
\hline (0.3 0.4$]$ & +0.003 & -0.845 & \pm 0.087 & \pm 0.272 \\
\hline (0.4 0.5 ) & -0.009 & -0.601 & 0.070 & 0.439 \\
\hline$\left(\begin{array}{lll}0.5 & 0.6\end{array}\right]$ & +0.001 & -0.100 & 0.056 & 0.291 \\
\hline (0.6 0.7 ( 0.7$]$ & -0.004 & +0.161 & 0.064 & 0.304 \\
\hline$\left(\begin{array}{lll}0.7 & 0.8\end{array}\right]$ & +0.003 & +0.200 & 0.062 & 0.250 \\
\hline (0.8 0.9 ) & 0.000 & +0.173 & 0.053 & 0.313 \\
\hline (0.9 1.0 & 0.000 & +0.048 & 0.034 & 0.197 \\
\hline (1.0 1.1$]$ & -0.001 & -0.385 & 0.063 & 0.288 \\
\hline
\end{tabular}

for UBV and Sloan photometries, and the new metallicity calibration for the Sloan photometric system

$$
\begin{aligned}
\left(g^{\prime}-r^{\prime}\right)_{0} & =1.05(B-V)_{0}-0.23 \\
\left(u^{\prime}-g^{\prime}\right)_{0} & =1.38(U-B)_{0}+1.14
\end{aligned}
$$

Let us write equation (9b) for two stars with the same $(B-V)_{0}$ (or equivalently $\left.\left(g^{\prime}-r^{\prime}\right)_{0}\right)$, i.e. for a Hyades star $(\mathrm{H})$ and for a star $(*)$ whose UV-excess is normalised to

$$
\begin{aligned}
\left(u^{\prime}-g^{\prime}\right)_{\mathrm{H}} & =1.38(U-B)_{\mathrm{H}}+1.14 \\
\left(u^{\prime}-g^{\prime}\right)_{*} & =1.38(U-B)_{*}+1.14
\end{aligned}
$$

Then, the UV-excess for the star in question, relative to the Hyades star is

$$
\left(u^{\prime}-g^{\prime}\right)_{\mathrm{H}}-\left(u^{\prime}-g^{\prime}\right)_{*}=1.38\left\lfloor(U-B)_{\mathrm{H}}-(U-B)_{*}\right\rfloor
$$

or, in standard notation

$$
\delta\left(u^{\prime}-g^{\prime}\right)=1.38 \delta(U-B)
$$

If we apply this equation to a star with $(B-V)_{0}=0.6$, corresponding to $\left(g^{\prime}-r^{\prime}\right)_{0}=0.4$, we obtain

$$
\delta\left(u^{\prime}-g^{\prime}\right)_{0.4}=1.38 \delta(U-B)_{0.6}
$$

for the relation between the normalised UV-excesses in the UBV and the Sloan systems. From this equation
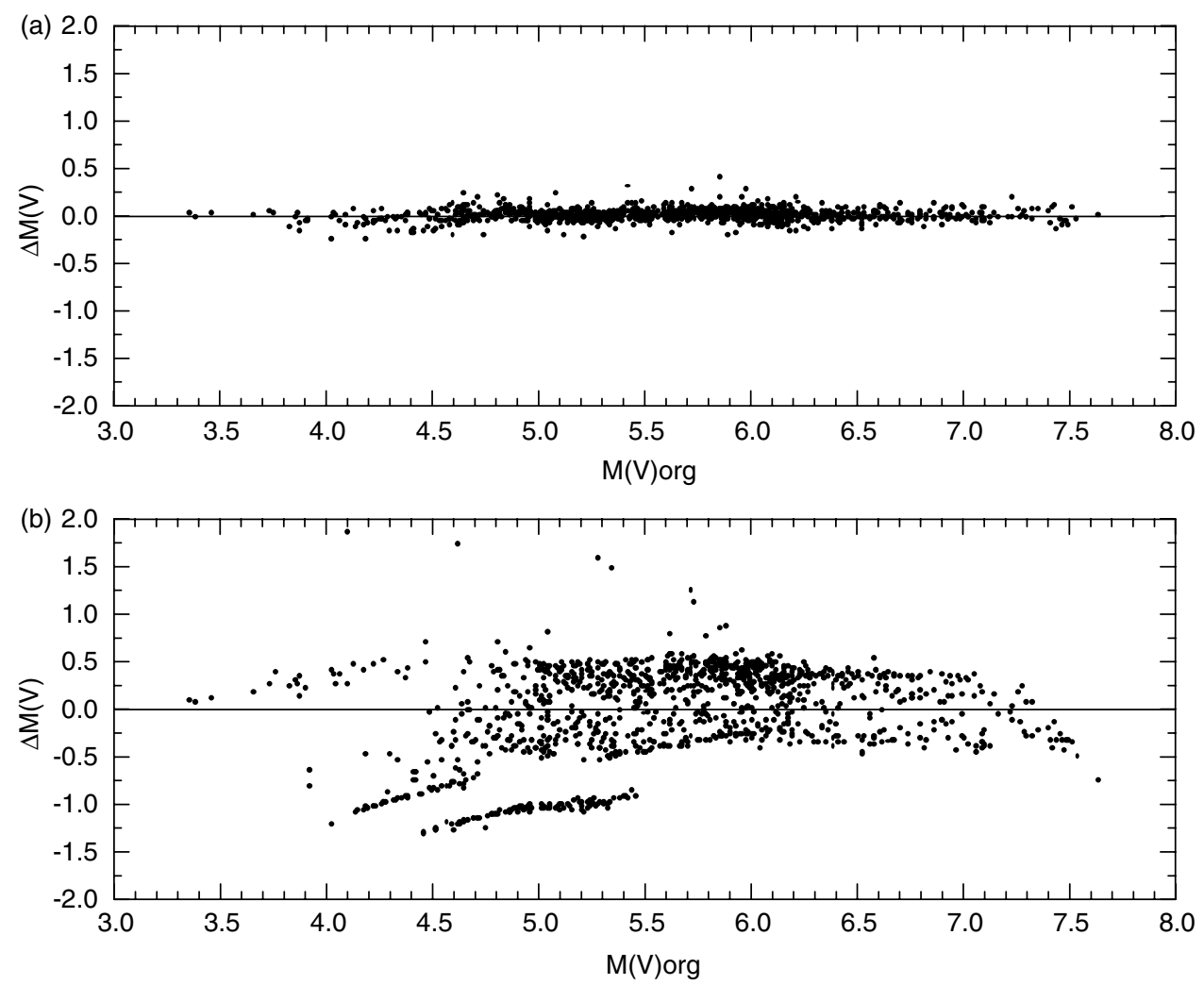

Figure 4 Deviation of the evaluated absolute magnitudes relative to the original absolute magnitudes for (a) the new procedure and (b) LCL. 
we obtain

$$
\delta(U-B)_{0.6}=0.725 \delta\left(u^{\prime}-g^{\prime}\right)_{0.4}
$$

which yields a new metallicity calibration for the Sloan photometry by its substitution in

$$
[\mathrm{Fe} / \mathrm{H}]=0.10-2.76 \delta_{0.6}-24.04 \delta_{0.6}^{2}+30.00 \delta_{0.6}^{3}
$$

which covers a large range of metallicity, i.e. $-2.75 \leq[\mathrm{Fe} / \mathrm{H}] \leq 0.2$ dex (Karaali et al., 2003). Hence, the new metallicity calibration for the Sloan photometry is obtained as follows

$$
[\mathrm{Fe} / \mathrm{H}]=0.10-2.00 \delta_{0.4}-12.64 \delta_{0.4}^{2}+11.43 \delta_{0.4}^{3}
$$

Finally, we can show that the coefficients given by Sandage (1969) for the UBV photometry can also be used for the normalisation of the UV-excesses in the Sloan photometry. Take another star with any $B-V$ (or equivalent $g^{\prime}-r^{\prime}$ ) but with the same metallicity as the first star. The relation between its normalised UV-excesses in the two systems would be as equation (12). Hence, from (12) and (13) we obtain

$$
\delta\left(u^{\prime}-g^{\prime}\right)_{0.4} / \delta\left(u^{\prime}-g^{\prime}\right)=\delta(U-B)_{0.6} / \delta(U-B)=f
$$

where $f$ is the $\mathrm{UV}$-excess normalised factor.

\subsection{Photometric Parallaxes for the Sloan Photometry}

As mentioned above, the procedure in Section 3.3 can be adopted for photometric parallax derivation also for the Sloan photometry by using the colour equations and the relation between the normalised UV-excesses in two systems. First, we draw $(B-V)_{0}$ from equation (9a)

$$
(B-V)_{0}=0.952\left(g^{\prime}-r^{\prime}\right)_{0}+0.219
$$

and then substitute it into (7) for normalisation of the Hyades main-sequence in the Sloan photometry as follows

$M_{g^{\prime}}^{\mathrm{H}}($ nor $)=-1.9330\left(g^{\prime}-r^{\prime}\right)_{0}^{2}+7.3742\left(g^{\prime}-r^{\prime}\right)_{0}+2.1036$

Bearing in mind that the offsets from the fiducial sequence of Hyades in two systems are equal, the offset for Sloan photometry can be derived by replacing the equivalence of $\delta(U-B)_{0.6}$ in equation (14) into (8). The following result is obtained:

$$
\Delta M_{g^{\prime}}^{\mathrm{H}}(\text { nor })=c_{3} \delta_{0.4}^{3}+c_{2} \delta_{0.4}^{2}+c_{1} \delta_{0.4}+c_{0}
$$

where the coefficients $c_{i}$ are given in Table 6 as a function of $\left(g^{\prime}-r^{\prime}\right)_{0}$.

\subsection{Comparison of the Absolute Magnitudes Derived by the New Procedure and the Colour-Magnitude Diagram of a Specific Cluster}

As an example, we compared the absolute magnitudes derived by the new procedure and the colour-magnitude diagram of cluster M13 used for the photometric paral-

\begin{tabular}{|c|c|c|c|c|}
\hline$\left(g^{\prime}-r^{\prime}\right)_{0}$ & $c_{3}$ & $c_{2}$ & $c_{1}$ & $c_{0}$ \\
\hline (0.09 0.19$]$ & -12.2631 & +8.3769 & +1.2579 & -0.0177 \\
\hline (0.19 0.30$]$ & -5.8617 & +1.9547 & +3.2516 & +0.0022 \\
\hline (0.30 0.40$]$ & +1.4904 & -2.5269 & +3.9039 & +0.0134 \\
\hline$\left(\begin{array}{lll}0.40 & 0.51]\end{array}\right.$ & -4.2566 & -0.1585 & +3.6454 & +0.0153 \\
\hline (0.51 0.61$]$ & +0.0400 & -1.9005 & +3.3492 & -0.0144 \\
\hline (0.61 0.72$]$ & -0.8588 & +0.0583 & +2.4990 & -0.0203 \\
\hline (0.72 0.82$]$ & -9.5159 & +3.8326 & +1.4695 & +0.0051 \\
\hline (0.82 0.93$]$ & -2.8210 & +2.2476 & +0.9163 & -0.0047 \\
\hline
\end{tabular}
lax estimation for halo dwarfs (cf. Chen et al. 2001). One
Table 6. Numerical values for the coefficients in equation (20) as a function of $\left(g^{\prime}-r^{\prime}\right)_{0}$ colour-index. The colour-index intervals correspond to the $(B-V)_{0}$ intervals in the first columns of Tables 2 and 4

Table 7. Colour-magnitude diagram for M13 in UBV and Sloan systems

\begin{tabular}{llll}
\hline$(B-V)_{0}$ & $M_{\mathrm{V}}$ & $\left(g^{\prime}-r^{\prime}\right)_{0}$ & $M_{g^{\prime}}$ \\
\hline 0.407 & 3.70 & 0.197 & 3.808 \\
0.410 & 3.90 & 0.201 & 4.010 \\
0.410 & 4.10 & 0.201 & 4.210 \\
0.419 & 4.30 & 0.210 & 4.415 \\
0.414 & 4.50 & 0.205 & 4.612 \\
0.440 & 4.70 & 0.232 & 4.826 \\
0.448 & 4.90 & 0.240 & 5.031 \\
0.500 & 5.10 & 0.295 & 5.260 \\
0.501 & 5.30 & 0.296 & 5.461 \\
0.531 & 5.50 & 0.328 & 5.677 \\
0.550 & 5.70 & 0.348 & 5.888 \\
0.587 & 5.90 & 0.386 & 6.109 \\
0.642 & 6.10 & 0.444 & 6.340 \\
0.682 & 6.30 & 0.486 & 6.562 \\
0.713 & 6.50 & 0.519 & 6.779 \\
0.784 & 6.70 & 0.593 & 7.019 \\
0.821 & 6.90 & 0.632 & 7.240 \\
0.864 & 7.10 & 0.677 & 7.464 \\
0.918 & 7.30 & 0.734 & 7.694 \\
0.945 & 7.50 & 0.762 & 7.909 \\
1.110 & 7.70 & 0.936 & 8.202 \\
\hline & & & \\
\hline
\end{tabular}

can extend this comparison to the other components of the Galaxy. The work is carried out as follows. First, we used the UBV data of Richer \& Fahlman (1986) and evaluated the $\left(g^{\prime}-r^{\prime}\right)_{0}$ and the $M_{g^{\prime}}$ absolute magnitudes for the main-sequence of M13 via equation (9a) and the following colour equation, which is adopted from Fukugita et al. (1996)

$$
M_{g^{\prime}}=M(V)+0.56(B-V)_{0}-0.12
$$

The $\left(g^{\prime}-r^{\prime}\right)_{0}$ and $M_{g^{\prime}}$ data thus obtained (Table 7) transform the main-sequence of cluster M13 from UBV to the Sloan photometry

$$
\begin{aligned}
M_{g}^{\prime}(\mathrm{M} 13)= & 11.442\left(g^{\prime}-r^{\prime}\right)_{0}^{3}-25.292\left(g^{\prime}-r^{\prime}\right)_{0}^{2} \\
& +21.599\left(g^{\prime}-r^{\prime}\right)_{0}+0.8621
\end{aligned}
$$

Equation (22) yields direct absolute magnitude estimates for metal-poor stars such as halo dwarfs. 
As a second step, we adopted seven sample of artificial stars with $\left(g^{\prime}-r^{\prime}\right)_{0}$ between 0.20 and 0.50 but with different metallicities, and evaluated their absolute magnitudes by using equation (19) and the related one in (20). The selection of this colour-index interval is due to the work of Chen et al. (2001). These authors assumed that stars fainter than $g^{\prime} \sim 18$ mag with $0.20 \leq\left(g^{\prime}-r^{\prime}\right)_{0} \leq 0.50$ belong to the halo population and used the colour-magnitude diagram of cluster M13, without any metallicity restriction, for their absolute magnitude determination. However, we adopted different metallicities for different samples to reveal the difference between two procedures. As it is easier to derive the metallicity from the normalised UV-excess, we adopted $\delta_{0.4}=0.00,0.10,0.20,0.30$, $0.40,0.50$ and 0.60 respectively which correspond to the metallicities $[\mathrm{Fe} / \mathrm{H}]=0.10,-0.21,-0.71,-1.33$, $-1.99,-2.63$ and -3.18 dex. Table 8 gives the full set of $\left(g^{\prime}-r^{\prime}\right)_{0}$ cited, the corresponding $M_{g^{\prime}}^{\mathrm{H}}($ nor $)$, the $\Delta M_{g^{\prime}}^{\mathrm{H}}$ (nor) and $M_{g^{\prime}}$.

Finally, we evaluated another set of $M_{g^{\prime}}$, by means of equation (22) and compared them with the $M_{g^{\prime}}$ in the seven sets mentioned above (Table 9). The mean of the differences between the $M_{g^{\prime}}$ derived by the new procedure and those evaluated by means of equation (22) i.e.: $<\Delta M>\equiv M_{g^{\prime}}(M 13)-M_{g^{\prime}}\left(\delta_{0.4}\right)$ are larger for relatively metal-rich stars as expected and least for $[\mathrm{Fe} / \mathrm{H}] \approx-2 \mathrm{dex}$. The following third-degree polynomial is a good fit to the couple $\left\langle\Delta M>\right.$ and $\delta_{0.4}$ (Figure 5)

$$
\begin{aligned}
\delta_{0.4}= & -0.2305<\Delta M>^{3}+0.5374<\Delta M>^{2} \\
& -0.6575<\Delta M>+0.4369
\end{aligned}
$$

Equation (23) also reveals that $\langle\Delta M\rangle=0$ for $\delta_{0.4}=0.4369$ or $[\mathrm{Fe} / \mathrm{H}]=-2.23 \mathrm{dex}$. This result indicates that a colour-magnitude diagram with metallicity less than the one for M13 $([\mathrm{Fe} / \mathrm{H}] \approx-1.4 \mathrm{dex})$ is more appropriate for the photometric parallax estimation for metal-poor stars in deep surveys such as SDSS (as explained in the discussion).

\section{Discussion}

We have used the high-precision UBV data of Ryan (1989) for absolute magnitude estimation. Although LCL already derived two equations, one for stars with metallicity $[\mathrm{Fe} / \mathrm{H}] \geq-1.75 \mathrm{dex}$ and another for extreme metal poor stars (equations 2 and 4 respectively), both equations are functions of $(B-V)_{0}$ and of the normalised $\delta_{0.6}$

Table 8. Absolute magnitudes for a set of artificial stars of different metallicities with $0.2 \leq\left(g^{\prime}-r^{\prime}\right)_{0} \leq 0.5$. The columns are (1) $\left(g^{\prime}-r^{\prime}\right)_{0}$ colour-index; (2) normalised absolute magnitude for a Hyades star of this colour-index; (3)-(9) and (10)-(16) absolute magnitude

\begin{tabular}{|c|c|c|c|c|c|c|c|c|c|c|c|c|c|c|c|}
\hline 1 & 2 & 3 & 4 & 5 & 6 & 7 & 8 & 9 & 10 & 11 & 12 & 13 & 14 & 15 & 16 \\
\hline ). 20 & 3.501 & 0.0022 & 0.341 & 0.684 & 0.995 & 1.240 & 1.384 & 1.391 & 3.483 & 3.842 & 4.185 & 4.496 & 4.742 & 4.885 & 4.892 \\
\hline 0.21 & 3.567 & 0.0022 & 0.341 & 0.684 & 0.995 & 1.240 & 1.384 & 1.391 & 3.549 & 3.908 & 4.251 & 4.562 & 4.807 & 4.951 & 4.958 \\
\hline 0.22 & 3.632 & 0.0022 & 0.341 & 0.684 & 0.995 & 1.240 & 1.384 & 1.391 & 3.615 & 3.973 & 4.316 & 4.628 & 4.873 & 5.016 & 5.023 \\
\hline 0.23 & 3.697 & 0.0022 & 0.341 & 0.684 & 0.995 & 1.240 & 1.384 & 1.3 & 3.680 & 4.038 & 4.381 & 4.693 & & 5.081 & 5.088 \\
\hline 0.24 & 3.762 & 0.002 & 0.341 & 0.684 & 0.995 & 1.2 & 1.384 & 1.3 & 3.744 & 4.1 & 4.446 & 757 & 03 & 146 & 5.153 \\
\hline 0.25 & 3.826 & 0.00 & 0.3 & 0.6 & 0.9 & 1.240 & 1.384 & 1.3 & & 4.167 & & & 667 & 210 & 5.217 \\
\hline 0.26 & 3.890 & 0.0022 & 0.341 & 0.684 & 0.995 & 1.240 & 1.384 & 1.391 & 3.873 & 4.231 & 4.574 & 4.886 & 5.131 & 5.274 & 5.281 \\
\hline 0.27 & 3.954 & 0.0022 & 0.341 & 0.684 & 0.995 & 1.240 & 1.384 & 1.391 & 3.936 & 4.295 & 4.638 & 4.949 & 5.194 & 5.338 & 5.344 \\
\hline 0.28 & 4.017 & 0.0022 & 0.341 & 0.684 & 0.995 & 1.240 & 1.384 & 1.391 & 3.999 & 4.358 & 4.701 & 5.012 & 5.257 & 5.401 & 5.408 \\
\hline 0.29 & 4.080 & 0.0022 & 0.341 & 0.684 & 0.995 & 1.240 & 1.384 & 1.391 & 4.062 & 4.421 & 4.763 & 5.075 & 5.320 & 5.464 & 5.470 \\
\hline 0.30 & 4.142 & 0.0022 & 0.341 & 0.684 & 0.995 & 1.240 & 1.384 & 1.391 & 4.124 & 4.483 & 4.826 & 5.137 & 5.382 & 5.526 & 5.533 \\
\hline 0.31 & 4.204 & 0.0134 & 0.380 & 0.705 & 0.997 & 1.266 & 1.520 & 1.767 & 4.217 & 4.584 & 4.909 & 5.201 & 5.470 & 5.724 & 5.970 \\
\hline 0.32 & 4.265 & 0.0134 & 0.380 & 0.705 & 0.997 & 1.266 & 1.520 & 1.767 & 4.279 & 4.645 & 4.970 & 5.263 & 5.531 & 5.785 & 6.032 \\
\hline 0.33 & 4.327 & 0.0134 & 0.380 & 0.705 & 0.997 & 1.266 & 1.520 & 1.767 & 4.340 & 4.707 & 5.032 & 5.324 & 5.593 & 5.847 & 6.093 \\
\hline 0.34 & 4.387 & 0.0134 & 0.380 & 0.705 & 0.997 & 1.266 & 1.520 & 1.767 & 4.401 & 4.767 & 5.092 & 5.385 & 5.653 & 5.907 & 6.154 \\
\hline 0.35 & 4.448 & 0.0134 & 0.380 & 0.705 & 0.997 & 1.266 & 1.520 & 1.767 & 4.461 & 4.828 & 5.153 & 5.445 & 5.714 & 5.968 & 6.214 \\
\hline 0.36 & 4.508 & 0.0134 & 0.380 & 0.705 & 0.997 & 1.266 & 1.520 & 1.767 & 4.521 & 4.888 & 5.213 & 5.505 & 5.774 & 6.028 & 6.274 \\
\hline 0.37 & 4.567 & 0.0134 & 0.380 & 0.705 & 0.997 & 1.266 & 1.520 & 1.767 & 4.581 & 4.947 & 5.272 & 5.565 & 5.833 & 6.087 & 6.334 \\
\hline 0.38 & 4.627 & 0.0134 & 0.380 & 0.705 & 0.997 & 1.266 & 1.520 & 1.767 & 4.640 & 5.007 & 5.332 & 5.624 & 5.893 & 6.147 & 6.393 \\
\hline 0.39 & 4.686 & 0.0134 & 0.380 & 0.705 & 0.997 & 1.266 & 1.520 & 1.767 & 4.699 & 5.066 & 5.391 & 5.683 & 5.952 & 6.205 & 6.452 \\
\hline 0.40 & 4.744 & 0.0134 & 0.380 & 0.705 & 0.997 & 1.266 & 1.520 & 1.767 & 4.757 & 5.1 & & & 6.010 & 6.264 & 6.511 \\
\hline 0.41 & 4.802 & 0.0153 & 0.374 & 0.704 & 0.980 & 1.176 & 1.266 & 1.224 & 4.817 & 5.176 & 5.506 & 5.782 & 5.978 & 6.068 & 6.026 \\
\hline 0.42 & 4.860 & 0.0153 & 0.374 & 0.704 & 0.980 & 1.176 & 1.266 & 1.224 & 4.875 & 5.234 & 5.564 & & .035 & 6.126 & 6.084 \\
\hline 0.43 & 4.917 & 0.0153 & 0.374 & 0.704 & 0.980 & 1.176 & 1.266 & 1.224 & 4.932 & 5.291 & 5.621 & 897 & .093 & 6.183 & 6.141 \\
\hline 0.44 & 4.974 & 0.0153 & 0.374 & 0.704 & 0.980 & 1.176 & 1.266 & 1.224 & 4.989 & 5.348 & 5.678 & 954 & 150 & 6.240 & 6.198 \\
\hline 0.45 & 5.031 & & 0.374 & 0.704 & 0.980 & 1.176 & 1.266 & 1.224 & 5.046 & 5.405 & 5.735 & 010 & 206 & 6.297 & 6.255 \\
\hline 0.46 & 5.087 & 0.0153 & 0.374 & 0.704 & 0.980 & 1.176 & 1.266 & 1.224 & 5.102 & 5.461 & 5.791 & 6.066 & 6.262 & 6.353 & 6.311 \\
\hline 0.4 & 5.142 & & 0.3 & 0.7 & 0.9 & 1.176 & 1.266 & 1.224 & & & & & & 6.409 & 6.366 \\
\hline 0.4 & 5.198 & & & 0.7 & 0.980 & 1.176 & 1.266 & 1.224 & 5.213 & & & & 6.374 & 6.464 & 6.422 \\
\hline 0.4 & 5.253 & & & 0.704 & 0.980 & 1.176 & 1.266 & 1.224 & & & & 6.233 & & 6.519 & 6.477 \\
\hline 0.50 & 5.307 & 0.0153 & 0.374 & 0.704 & 0.980 & 1.176 & 1.266 & 1.224 & 5.323 & 5.681 & 6.011 & 6.287 & 6.483 & 6.574 & 6.531 \\
\hline
\end{tabular}
differences $\left(\Delta M_{g^{\prime}}^{\mathrm{H}}(\right.$ nor $\left.)\right)$ and absolute magnitudes $M_{g^{\prime}}$ evaluated for $\delta_{0.4}=0.0,0.1,0.2,0.3,0.4,0.5$ and 0.6 respectively 
Table 9. Comparison of the absolute magnitudes estimated by the new procedure and by means of a colour-magnitude diagram for the cluster M13 for the artificial stars in question. The columns are (1) colour index $\left(g^{\prime}-r^{\prime}\right)_{0} ;(2)$ absolute magnitude $M_{g^{\prime}}$ (M13) evaluated by equation (22); (3)-(9) difference between the absolute magnitude $M_{g^{\prime}}$ (M13) and the absolute magnitudes estimated for $\delta_{0.4}=0.0-0.6($ columns 10-16, Table 8). The averages of these differences $(<\Delta M>)$ and the corresponding standard deviations $\sigma$ are given beneath

\begin{tabular}{|c|c|c|c|c|c|c|c|c|}
\hline 1 & 2 & 3 & 4 & 5 & 6 & 7 & 8 & 9 \\
\hline 0.20 & 4.260 & 0.776 & 0.418 & 0.075 & -0.237 & -0.482 & -0.625 & -0.632 \\
\hline 0.21 & 4.386 & 0.837 & 0.478 & 0.136 & -0.176 & -0.421 & -0.565 & -0.571 \\
\hline 0.22 & 4.509 & 0.895 & 0.536 & 0.193 & -0.118 & -0.363 & -0.507 & -0.514 \\
\hline 0.23 & 4.629 & 0.949 & 0.590 & 0.248 & -0.064 & -0.309 & -0.453 & -0.459 \\
\hline 0.24 & 4.745 & 1.000 & 0.642 & 0.299 & -0.013 & -0.258 & -0.401 & -0.408 \\
\hline 0.25 & 4.857 & 1.049 & 0.690 & 0.347 & 0.036 & -0.209 & -0.353 & -0.360 \\
\hline 0.26 & 4.967 & 1.094 & 0.735 & 0.393 & 0.081 & -0.164 & -0.308 & -0.314 \\
\hline 0.27 & 5.073 & 1.137 & 0.778 & 0.435 & 0.124 & -0.122 & -0.265 & -0.272 \\
\hline 0.28 & 5.175 & 1.176 & 0.817 & 0.475 & 0.163 & -0.082 & -0.225 & -0.232 \\
\hline 0.29 & 5.275 & 1.213 & 0.854 & 0.512 & 0.200 & -0.045 & -0.189 & -0.195 \\
\hline 0.30 & 5.371 & 1.247 & 0.889 & 0.546 & 0.234 & -0.011 & -0.154 & -0.161 \\
\hline 0.31 & 5.465 & 1.248 & 0.881 & 0.556 & 0.264 & -0.005 & -0.259 & -0.505 \\
\hline 0.32 & 5.556 & 1.277 & 0.910 & 0.585 & 0.293 & 0.024 & -0.230 & -0.476 \\
\hline 0.33 & 5.643 & 1.303 & 0.937 & 0.612 & 0.319 & 0.051 & -0.203 & -0.450 \\
\hline 0.34 & 5.728 & 1.328 & 0.961 & 0.636 & 0.344 & 0.075 & -0.179 & -0.426 \\
\hline 0.35 & 5.811 & 1.349 & 0.983 & 0.658 & 0.365 & 0.097 & -0.157 & -0.404 \\
\hline 0.36 & 5.890 & 1.369 & 1.002 & 0.677 & 0.385 & 0.116 & -0.138 & -0.384 \\
\hline 0.37 & 5.967 & 1.386 & 1.020 & 0.695 & 0.402 & 0.134 & -0.120 & -0.367 \\
\hline 0.38 & 6.042 & 1.402 & 1.035 & 0.710 & 0.418 & 0.149 & -0.105 & -0.352 \\
\hline 0.39 & 6.114 & 1.415 & 1.048 & 0.723 & 0.431 & 0.162 & -0.092 & -0.339 \\
\hline 0.40 & 6.183 & 1.426 & 1.059 & 0.734 & 0.442 & 0.173 & -0.081 & -0.327 \\
\hline 0.41 & 6.251 & 1.433 & 1.075 & 0.745 & 0.469 & 0.273 & 0.182 & 0.225 \\
\hline 0.42 & 6.316 & 1.441 & 1.082 & 0.752 & 0.476 & 0.280 & 0.190 & 0.232 \\
\hline 0.43 & 6.379 & 1.446 & 1.088 & 0.758 & 0.482 & 0.286 & 0.195 & 0.238 \\
\hline 0.44 & 6.439 & 1.450 & 1.091 & 0.761 & 0.486 & 0.290 & 0.199 & 0.241 \\
\hline 0.45 & 6.498 & 1.452 & 1.094 & 0.764 & 0.488 & 0.292 & 0.201 & 0.244 \\
\hline 0.46 & 6.555 & 1.453 & 1.094 & 0.764 & 0.489 & 0.293 & 0.202 & 0.244 \\
\hline 0.47 & 6.610 & 1.452 & 1.093 & 0.763 & 0.488 & 0.292 & 0.201 & 0.243 \\
\hline 0.48 & 6.663 & 1.450 & 1.091 & 0.761 & 0.485 & 0.289 & 0.199 & 0.241 \\
\hline 0.49 & 6.714 & 1.446 & 1.087 & 0.757 & 0.482 & 0.286 & 0.195 & 0.237 \\
\hline \multirow[t]{3}{*}{0.50} & 6.764 & 1.441 & 1.082 & 0.752 & 0.477 & 0.281 & 0.190 & 0.232 \\
\hline & $<\Delta M>$ & 1.269 & 0.908 & 0.575 & 0.281 & 0.044 & -0.118 & -0.186 \\
\hline & $\sigma$ & 0.205 & 0.204 & 0.210 & 0.224 & 0.237 & 0.256 & 0.313 \\
\hline
\end{tabular}

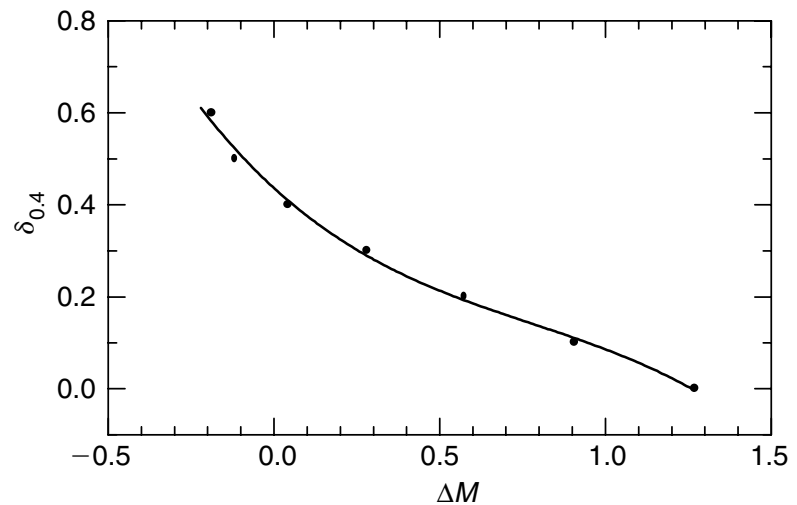

Figure $5 \delta_{0.4}$ versus mean absolute magnitude difference $\langle\Delta M\rangle$.

UV-excess. However, as it can be seen from Figures 1 and 3 , the offset from the fiducial main-sequence of Hyades behaves differently for different colour-index intervals, confirming the necessity of different equations for different $(B-V)_{0}$ intervals.
As admitted by LCL, they forced their calibration in order to pass through the zero point, thus supplying the Hyades absolute magnitudes for $\delta_{0.6}=0$. In this study we have used the updated data (see Appendix) and have obtained a quadratic equation for the Hyades sequence. However, our calibration does not pass through the zero point either. Hence, we normalised the fiducial main-sequence of Hyades. This approach supplies absolute magnitudes almost equal to the Hyades absolute magnitudes for $\delta_{0.6}=0$, for all $(B-V)_{0}$ intervals.

The comparison of the estimated absolute magnitudes with the original ones confirms the accuracy of our calibration. The mean of the differences of absolute magnitudes for each $(B-V)_{0}$ interval is almost zero and their standard deviations are only few percent (Table 5). The mean difference for stars with $0.3<(B-V)_{0} \leq 1.1$ and the corresponding standard deviation are +0.0002 and \pm 0.0613 mag, respectively. Moreover, the plot of these differences versus the original absolute magnitudes shows that most of the stars lie within the interval $-0.1<\Delta M(V)<+0.1$ 
(Figure 4a). Whereas the comparison of the absolute magnitudes estimated by LCL with the original ones gives larger means and standard deviations (Table 5). The mean difference and the corresponding mean standard deviation for all stars are -0.0151 and \pm 0.4782 mag, respectively, rather different values than those from the new procedure. Finally, Figure $4 \mathrm{~b}$ also demonstrates the large range of the absolute magnitude differences for LCL, i.e. the majority of stars lie within $-0.5<\Delta M(V)<+0.5$ and there are about one hundred stars with still larger differences.

The colour-equations of Fukugita et al. (1996) provide a new metallicity calibration for the Sloan photometry. This has been carried out by the relation of normalised UV-excesses in the UBV and Sloan photometric systems, i.e. by substituting $\delta(U-B)_{0.6}=0.725 \delta\left(g^{\prime}-r^{\prime}\right)_{0.4}$ into the metallicity calibration of Karaali et al. (2003). The same substitution into equation (8) transforms the offset from the fiducial main-sequence of Hyades from UBV to Sloan photometry (equation 20) and finally the combination of (19) and (20) provides absolute magnitude estimation for the Sloan photometry.

We applied the new procedure to a set of artificial stars with $\left(g^{\prime}-r^{\prime}\right)_{0}$ between 0.20 and 0.50 , and compared the absolute magnitudes derived for seven different metallicities with the absolute magnitudes evaluated by means of the colour-magnitude diagram of M13. This is an example to see how coincident are the present approach and the canonical one. The mean of the differences between the absolute magnitudes derived by the new procedure and the canonical one is large for relatively metal-rich stars, is zero for the metallicity $[\mathrm{Fe} / \mathrm{H}]=-2.23 \mathrm{dex}$ and has a large range extending from $\langle\Delta M\rangle=1.269$ to $<\Delta M>=-0.186$. It is surprising that the coincidence occurs for the metallicity of M92 but not for the metallicity of M13 $([\mathrm{Fe} / \mathrm{H}]=-1.4 \mathrm{dex})$. One can argue that the metalrich stars are not efficient in the deep surveys. However, the range of $\langle\Delta M\rangle$ extends from +0.4 to -0.2 even for the metallicity range from -1.0 to $-3.0 \mathrm{dex}$, which is dominated by Population II stars. Additionally, the standard deviations (Table 9) for the seven comparisons mentioned above are larger than $\sigma= \pm 0.2 \mathrm{mag}$, resulting in an extra internal error in absolute magnitude estimation. The combination of these effects encourages us to claim that a single colour-magnitude diagram does not supply reliable absolute magnitudes for stars with a large range of metallicities. On the other hand, the small scattering of the differences between the original and the estimated absolute magnitudes for the UBV photometry confirms the significant improvement of the new procedure with respect to that of LCL. Finally, regarding the colour-equations of Fukugita et al. (1996), we argue that the new procedure can also be applied extensively and efficiently to SDSS (and to other systems, using appropriate colour-equations).

\section{Accessory Materials}

An Appendix detailing a Hyades sequence evolution and the raw data (Tables S1 and S2) are available as accessory material from PASA (www.csiro.au/journals/pasa/) or from the authors.

\section{References}

Cameron, L. M. 1985, 146, 59

Carney, B. W. 1979, ApJ, 233, 211

Chen, B., Stoughton, C., Smith, J. A., Uomoto, A., Pier, J. R, Yanny, B., Ivezic, Z., York, D.G., Anderson, J. E., \& Annis, J. 2001, ApJ, 553, 184

ESA 1997, The Hipparcos and Tycho Catalogues, ESA SP-1200

Fukugita, M., Ichikawa, T., Gunn, J. E., Doi, M., Shimasaku, K., \& Schneider, D. P. 1996, AJ, 111, 1748

Karaali, S., Bilir, S., Karatas, Y., \& Ak, S. G. 2003, PASA, 20, 165

Laird, J. B., Carney, B. W., \& Latham, D. W. 1988, AJ, 95, 1843 (LCL)

Nissen, P. E., \& Schuster, W. J. 1991, A\&A, 251, 457

Perryman, M. A. C., Brown, A. G. A., Lebreton, Y., Gomez, A., Turon, C., de Strobel, G. Cayrel; Mermilliod, J. C., Robichon, N., Kovalevsky, J., Crifo, F. 1998, A\&A, 331, 81

Phleps, S., Meisenheimer, K., Fuchs, B., \& Wolf, C. 2000, A\&A, 356, 108

Richer, H. B., \& Fahlman, G. G. 1986, ApJ, 304, 273

Ryan, S. G. 1989, AJ, 98, 1693

Sandage, A. 1969, ApJ, 158, 1115

Siegel, M. H., Majewski, S. R., Reid, I. N., \& Thompson, I. B. 2002 , AJ, 578, 151

Yi, S., Demarque, P., Kim, Y., Lee, Y., Ree, C. H., Lejeune, T., Barnes, S. 2001, ApJS, 136, 417 